ra», Trahajos Varios del SIP, núm. 89, págs. 639-661, Valencia.

HAYFs, J. W., 1968: «Excavations at Saraçhane in Istambul.Fifth Preliminary Report», Dumharton Oaks Papers, XXII, págs. 194216, Washington D.C.

Hayes, J.W., 1971: «A New Type of Early Christian Ampullan, British School at Athens, vol. 66. págs. 243-248.

LOFFREDA, S., 1985: Lucerne Bizantine in Terra Santa con Inscrizioni in Greco, Studium Biblicum Franciscanum, Collectio Maior, n. ${ }^{\circ} 35$, Jerusalén.

Lusuardi, S., y Murialdo, G., 1991: «Le ceramiche mediterranee in Liguria durante il periodo bizantino (VI-VII secolo)", págs. 123 146, en: A cerámica medieval no Mediterrâneo occidental, Actas do IV Congresso Internacional, Lisboa, 16-22 de Novembro 1987.

Maginiss, J., 1989: A Typology of the Late Roman and Byzantine Pottery of Jerusalem, University of Pennsylvania, University Microfilms International, Ann Arbor (1991), 2 vols.

Matfu y Llopis, F., 1972: «Bronces romanos imperiales y vándalos en l'Illa de Cullera», Archivo de Prehistoria Levantina, XIII, págs. 241-256, Valencia.

Parman, E., 1989: «The Pottery from St. John's Basilica at Ephesos», págs. 277-289, en:
Recherches sur la Céramique Bvaantine, Bulletin de Correspondance Hellénique, suppl. XVIII, École Française d'Athènes.

RILI:Y, J., 1975: «The Pottery from the First Session of Excavation in the Caesarea Hippodrome», Bulletin of the American Schools of Oriental Research. 218 , págs. 25-63.

Robinson, H. S., 1959: Pottery of the Roman Period. The Athenian Agora, vol. 5, American School of Clasical Studies at Athens, Princeton.

Rossflló, M., (en prensa): «Punta de l'llla de Cullera (Valencia): Un posible establecimiento monástico del s. vi d. C.n, IV Reunió d'Arqueologia Cristiana Hispànica, 28 septiembre/2 de octubre, 1992, Lisboa.

Salle: S. J., 1957: Excavations at Bethany (1949-1953), Studium Biblicum Franciscanum, 12, Jerusalén.

Tushingham, A. D., 1972: The Excavations at Dibon (Dhibân) in Moab. Third Campaign. 1952-53, Annual of the American Schools of Oriental Research, 40.

Tushingham, A. D., 1985: Excavations in Jerusalem, 1961-1967, vol. I, Royal Ontario Museum, Toronto.

Williams, D. F., 1983: «The Petrology of Certain Byzantine Amphorae: Some Suggestions as to Origins», págs. 99-110, en: Actes du colloque sur la céramique antique, (Carthage, 23-24 juin 1980), Dossier 1, CEDAC.

\title{
UN TIPO DE ÁNFORA TARDO-ROMANA POCO CONOCIDO (VLR 8.198)
}

\author{
POR \\ JOSEP ANTON REMOLÀ VALLVERDÚ \\ CODEX sccl-Arqueologia i Patrimoni
}

RESUMEN

El estudio de las ánforas procedentes de diversos depósitos bajo-imperiales (siglo $v$ d. C.) excavados recientemente en Tarragona permite caracterizar un tipo de ánfora poco conocido (VLR 8.198), de origen posiblemente oriental, cuya presencia en estos contextos tarraconenses representa un $2,6 \%$ sobre el número total estimado de ejemplares de ánfora (606).

\section{SUMMARY}

The study of amphorae from several late Roman contexts (fifth century $A D$ ), recently excavated at Tarragona, allows to characterize an unknown type (VLR 8.198), possibly originated in Eastern Mediterranean. It comprises $2,6 \%$ of all estimated samples (606). 


\section{INTRODUCCIÓN}

Durante el estudio de las ánforas recuperadas en la excavación de la escombrera tardo-romana de la calle Vila-roma de Tarragona (1987), datada en el segundo cuarto avanzado del siglo $\vee$ d. C., detectamos la presencia de diversos fragmentos de ánfora que presentaban unas características físicas y tipológicas muy homogéneas, que no fue posible adscribir a ninguno de los tipos definidos con anterioridad, por lo que se incluyeron en el apartado de tipos indeterminados con la numeración 8.198-8.205 (Remolà/Abelló, 1989, 249-320; TED'A, 1989 a).

Posteriormente, esta presencia se observó en otros contextos tarraconenses de cronologia similar: Calle Vila-roma (1986), calle Trinquet Vell 4 (1989), Hospital vell de Santa Tecla (1990), Plaza dels Angels (1986) y Antiga Audiència (1986). En todos ellos, la presencia de este tipo --que continuaremos denominando con las siglas VLR 8.198- es numéricamente baja, la cronología de los depósitos analizados se sitúa dentro del siglo $\mathrm{v} y$, a pesar de ligeras variaciones formales y técnicas, el conjunto de fragmentos localizados mantiene una similitud que nos permite individualizar y caracterizar el tipo.

Considerando estas premisas, hemos creido conveniente presentar, aunque sea parcialmente, los fragmentos hasta ahora localizados para contribuir al conocimiento de este tipo en particular y al estudio general de las ánforas de este periodo. Incluimos, también, los resultados que, referidos a este tipo, se obtuvieron a partir de los análisis químicos realizados con muestras procedentes de la escombrera de la calle Vila-roma (1987).

\section{CALLE VILLA-ROMA 1986-1987}

En 1986, se realizó una primera intervención de urgencia en un solar de la parte alta de Tarragona, comprendido entre las calles Cuirateries, Natzaret y Vila-roma', espacio que correspondía al ángulo sud-oriental de la plaza

1 La dirección técnica corrió a cargo de J. A. Remolà y X. Dupré. de representación del foro provincial (figura 1). El resultado fue la localización de diversas estructuras de época romana y medieval y de una escombrera del siglo $v$ d. C. (básicamente, UE 206 y 306), excavada en los rellenos constructivos del recinto foral. El año siguiente, el Taller-Escola d'Arqueologia de Tarragona (TED’A) excavó en extensión la escombrera (UE 918), recuperándose un total de 30.549 fragmentos cerámicos que fueron objeto de estudio (TED’A, 1989 a). La cronologia de la escombrera se situó en el segundo cuarto avanzado del siglo $v$ d. C.

El total estimado de ejemplares anfóricos es de 400: 102 de origen oriental $(25.5 \%), 100$ de origen sudhispánico $(25 \%), 98$ de procedencia africana $(24,4 \%)$ y 21 de posible origen tarraconense $(5,2 \%)$. Los restantes 79 ejemplares $(19,7 \%)$ corresponden a un grupo heterogéneo que incluye ánforas de origen no determinado (figura 7). El tipo VLR 8.198 se incluyó en este último grupo dada su indeterminación de origen. El número estimado de ejemplares de este tipo es de ocho, lo cual significa un $2 \%$ sobre el total estimado de ánforas ${ }^{2}$. Además de estos ejemplares, la anterior intervención, realizada el año 1986, proporcionó un total de cinco fragmentos atribuibles a este tipo, que no se incluyen en el estudio cuantitativo, aunque si en el inventario.

En el marco de un acuerdo de colaboración entre el TED'A y el Departamento de Química Analítica de la Delegación en Tarragona de la Universidad de Barcelona (actual Universitat Rovira i Virgili) se realizó un análisis de 85 muestras de ánfora por florescencia de rayos-X del contenido en hierro, manganeso, titanio, calcio, potasio, silicio, aluminio, fósforo y magnesio, expresado como porcentajes de sus respectivos óxidos. El objetivo era obtener una caracterización química de las muestras para su ulterior análisis mediante la aplicación del método de Ward (análisis de agrupaciones jerarquizadas) y el de componentes principales (análisis de representación), con el objeto de

\footnotetext{
2 Excluimos el fragmento 8.203, el cual tras un análisis más contrastado consideramos de muy dudosa adscripción a este tipo.
} 


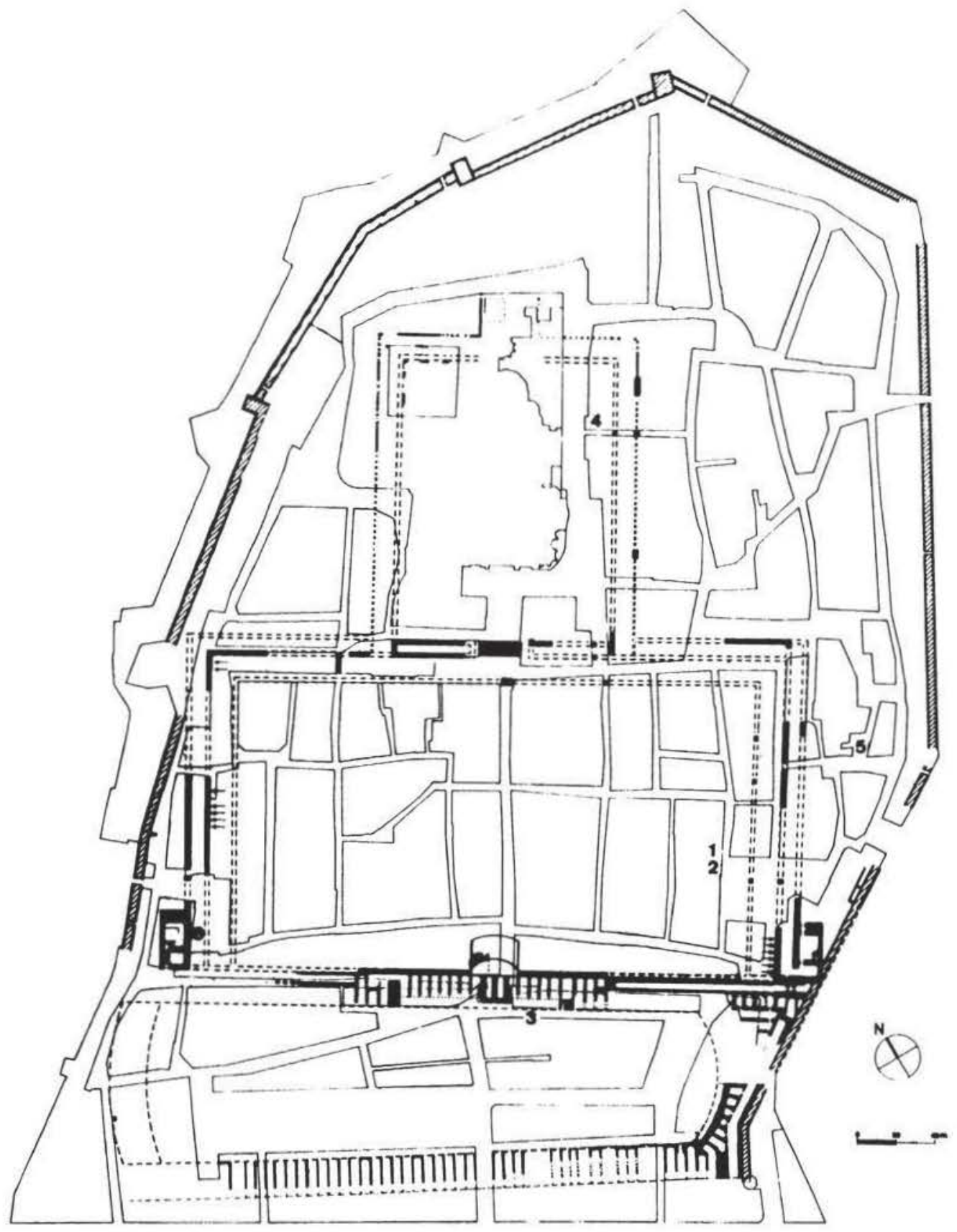

Figura 1.-Planta de las murallas romanas y del complejo de ceremonias provincial, superpuesta a la trama urbana actual, con indicación de las intervenciones arqueológicas analizadas (a partir de TED'A,1989): [1] Vila-roma 1986, [2] Vila-roma 1987, [3] Trinquet Vell-4, [4] Hospital Vell de Santa Tecla, [5] Plaza dels Angels y [6] Antiga Audiència. 
estudiar las agrupaciones naturales del conjunto de 66 muestras con origen conocido y, posteriormente, a través del método SIMCA, evaluar la pertenencia de las 19 muestras extraidas de ánforas de origen no determinado a alguna de las zonas de procedencia ya establecida ${ }^{3}$

De las muestras analizadas, cuatro correspondian al tipo VLR 8.198 (figura 6). Se constató una gran homogeneidad química de estas muestras, con niveles de similitud máximos en el diagrama de Ward, que constituian una agrupación natural diferenciada del resto de agrupaciones formadas por ánforas de procedencia determinada. Este hecho no nos permite relacionar, a nivel de composición quimica, el tipo VLR 8.198 con ánforas de procedencia conocida, lo cual nos hubiera permitido disponer de un dato objetivo para plantear su origen.

\section{INVENTARIO}

1. Parte superior. Número de registro: VLR-918-2975. Diámetro de abertura de la boca: $6,4 \mathrm{~cm}$. Bibliografia: Remolà/Abelló, 1989, 304305, fig. 165-8.198 (Figura 2).

2. Parte superior. Número de registro: VLR-918-2977. Diámetro de abertura de la boca: $6,2 \mathrm{~cm}$. Presenta restos muy fragmentarios y deteriorados de un posible titulus pictus en tinta roja situados entre el cuello y el hombro. $\mathrm{Bi}$ bliografia: Remolà/Abelló, 1989, 304-305, fig. 165-8.199 (Figura 2).

3. Fragmento de borde. Número de registro: VLR-918-2970. Diámetro de abertura de la boca: $6 \mathrm{~cm}$. Bibliografia: Remolà/Abelló, 1989, 304-305, fig. 165-8.200 (Figura 2).

4. Fragmento de borde. Número de registro: VLR-918-2974. Diámetro de abertura del borde: $6,2 \mathrm{~cm}$. Bibliografia: Remolà/Abelló, 1989, 304-305, fig. 165-8.201 (Figura 2).

5. Fragmento de borde. Número de registro: VLR-918-2971. Diámetro de abertura de

\footnotetext{
3 Un artículo con los primeros resultados ha sido enviado a la revista TALANTA. The International Journal of Pure and Applied Analytical Chemistry. J.A. Remolà, $X$. Rius, M. Larrechi: 5 th Century $\mathrm{AD}$ amphora producing centers in the Mediterranean. Chemometrycal characterization.
}
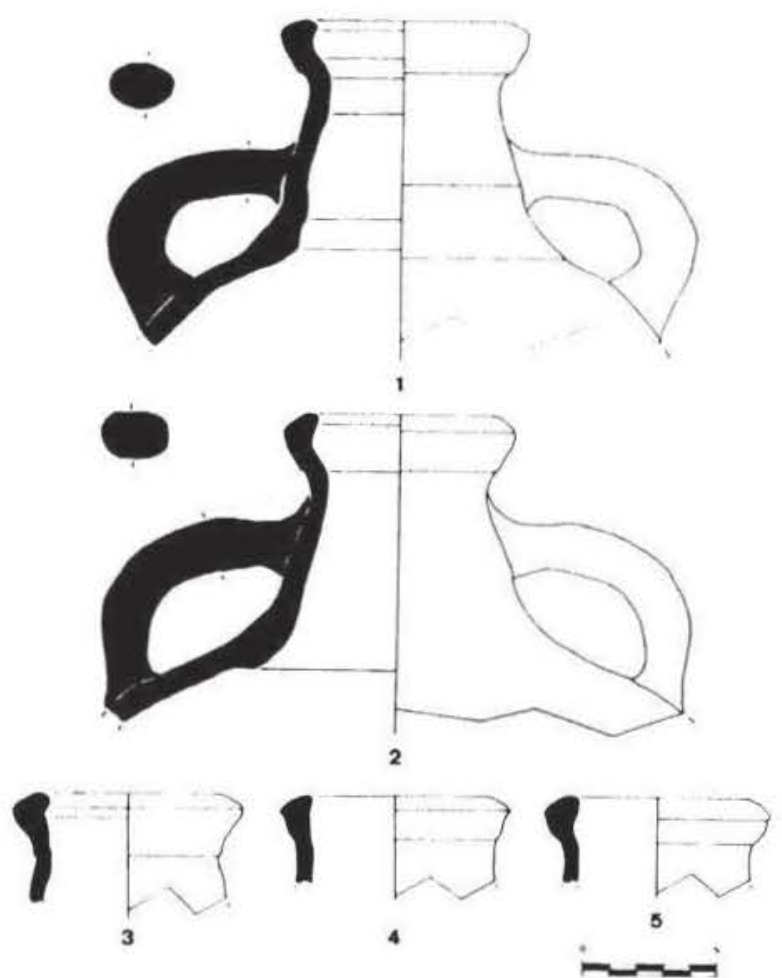

Figura 2.-Fragmentos procedentes de la intervencion de Vila-roma (1987) [1-5]. E. 1:4.

la boca: $6 \mathrm{~cm}$. Bibliografía: Remolà/Abelló, 1989, 304-305, fig. 165-8.202 (Figura 2).

6. Fragmento de base. Número de registro: VLR-918-2980. Bibliografia: Remolà/Abelló 1989 , págs. $304-305$, fig. 165-8.204 (figura 3).

7. Fragmento de base. Número de registro: VLR-918-2981. Bibliografia: Remolà/ Abelló, 1989, 304-305, fig. 165-8.205 (Figura 3 ).

8. Fragmento de borde. Número de Registro: 861-206-8662. Diámetro de abertura de la boca: $5,6 \mathrm{~cm}$ (Figura 3).

9. Fragmento de borde. Número de registro: $861-201-3127 / 3129 / 3130 / 3131 / 3132$. Diámetro de abertura de la boca: $6 \mathrm{~cm}$. (Figura 3).

10. Fragmento de borde. Número de registro: 86I-201-3128. Diámetro de abertura de la boca: $6 \mathrm{~cm}$ (Figura 3).

11. Fragmento de borde. Número de registro: 86I-206-8661 (Figura 3). 


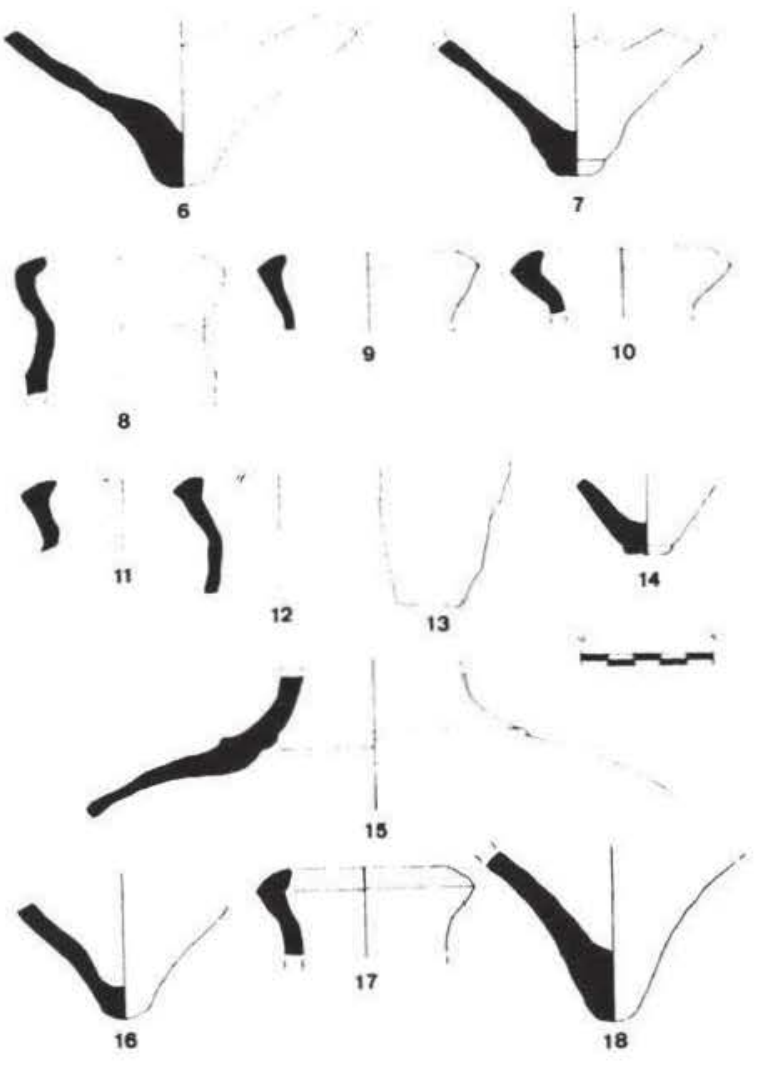

Figura 3,-Fragmentos procedentes de la intervención de Vila-roma (1987) [6-7], Vila-roma (1986) [8-11], Trinquet Vell-4 (1989) [12-16] y Hospital Vell de Santa Tecla $(1990)[17-18]$. E. 1:4

\section{CALLE TRINQUET VELL-4, 1989}

En 1989, el Taller-Escola d'Arqueologia de Tarragona (TED'A) realizó una intervención arqueológica de urgencia delante del inmueble número 4 de la calle Trinquet Vell, afectando un espacio ocupado en época alto-imperial por la arena del Circo (figura 1). Por encima de la cota conservada de los rellenos que constituyen la arena, se detectó la presencia de un potente nivel interpretado como escombrera (UEs $106,110,111,113$ y 114), cuya cronologia se sitúa dentro del siglo $v$ d. C., posiblemente en un momento avanzado del mismo (Aquilué, 1992, 801).

El número estimado de ánforas es de 93 : 31 de origen africano $(33,3 \%), 25$ de procedencia oriental $(26,8 \%), 15$ de origen sudhispánico $(16,1 \%)$ y dos de posible origen tarraconense
$(2,1 \%)$. Los 20 ejemplares restantes $(21,5 \%)$ son de origen no determinado (figura 7). El tipo VLR 8.198 está presente con tres ejemplares, lo cual representa un $2,1 \%$ sobre el total estimado de ánforas recuperadas.

\section{INVENTARIO}

12. Fragmento de borde. Número de registro: CTV/4-106-225 (Figura 3).

13. Fragmento de pared con restos fragmentarios de titulus pictus en tinta roja. Número de registro: CTV/4-106-223 (Figura 3).

14. Fragmento de base. Número de registro: CTV/4-106-226. Base apuntada con pivote poco prominente que presenta un pequeño resalte en la base (Figura 3 ).

15. Fragmento de cuello. Número de registro: CTV/4-106-227. Presenta restos muy deteriorados de un posible titulus pictus en tinta roja. (Figura 3).

16. Fragmento de base. Número de registro: CTV/4-110-62. Base apuntada con pivote poco prominente (Figura 3 ).

\section{HOSPITAL VELL DE SANTA TECLA, 1990}

En 1989, se realizó una primera intervención de urgencia en el interior del Hospital Vell de Santa Tecla por parte del Taller-Escola d'Arqueologia de Tarragona (TED'A), cuyos resultados a nivel anfórico no incluimos aquí por estar en fase de estudio. Un año más tarde, se efectuó una segunda intervención a cargo del Centro municipal de arqueología de Tarragona ${ }^{4}$, cuyos resultados son los que presentamos. Ambas intervenciones afectaban una escombrera bajo-imperial situada en el pórtico occidental del recinto de culto del gran complejo urbanístico alto-imperial, parcialmente reutilizado para la construcción del Hospital de Santa Tecla en el siglo XII (figura 1).

La cronología de la escombrera se sitúa en la primera mitad del siglo v (Aquilué, 1992,

\footnotetext{
4 Excavación dirigida por P. Pujante, al cual agradecemos el haber puesto a nuestra disposición los materiales, y A. Dasca.
} 
802 ), recuperándose un total de 2370 fragmentos cerámicos, 1829 de los cuales correspondian a ánforas. El total estimado de ánforas es de 45 que, en función de su área de producción, se distribuyen de la siguiente manera: 10 cuyo origen se sitúa en el Mediterráneo Oriental $(22.2 \%)$, ocho procedentes del Norte de A frica $(17,7 \%)$, siete originarias del Sur de Hispania $(15,5 \%)$, cuatro de posible origen tarraconense $(8,8 \%)$ y 16 de procedencia no determinada $(35,5 \%)$ (figura 7 ). El número estimado de ejemplares pertenenciente al tipo VLR 8.198 es de dos y representa, por tanto, el $4,4 \%$ sobre el total estimado de ánforas.

\section{INVENTARIO}

17. Fragmento de borde. Número de registro: HST-90-109-171. Diámetro de abertura de la boca: $5,6 \mathrm{~cm}$ (Figura 3 ).

18. Fragmento de base. Número de registro: HST-90-109-318. Presenta un pequeño pivote macizo (Figura 3 ).

19. Parte superior. Número de registro: HST-90-109-172/297. Diámetro de abertura de la boca: $5,8 \mathrm{~cm}$ (Figura 4).

\section{PLAZA DELS ANGELS, 1986}

En 1986, la remodelación de la plaza dels Angels, ubicada entre el muro oriental de cierre del pórtico de la plaza de representación del foro y el paramento interior de la muralla tardo-republicana (figura 1), propició una intervención arqueológica que permitió documentar los restos de una posible escombrera bajoimperial excavada en los rellenos constructivos de época alto-imperial (básicamente, UE 223 y $224)^{5}$. La cronología ha de situarse de forma genérica, por el escaso material datable recuperado, dentro del siglo $\mathrm{v}$, sin mayores precisiones.

Analizando conjuntamente las unidades estratigráficas 223 y 224 , de cronología aparen-

s Intervención arqueológica dirigida por J. A. Remolà y X. Dupré.

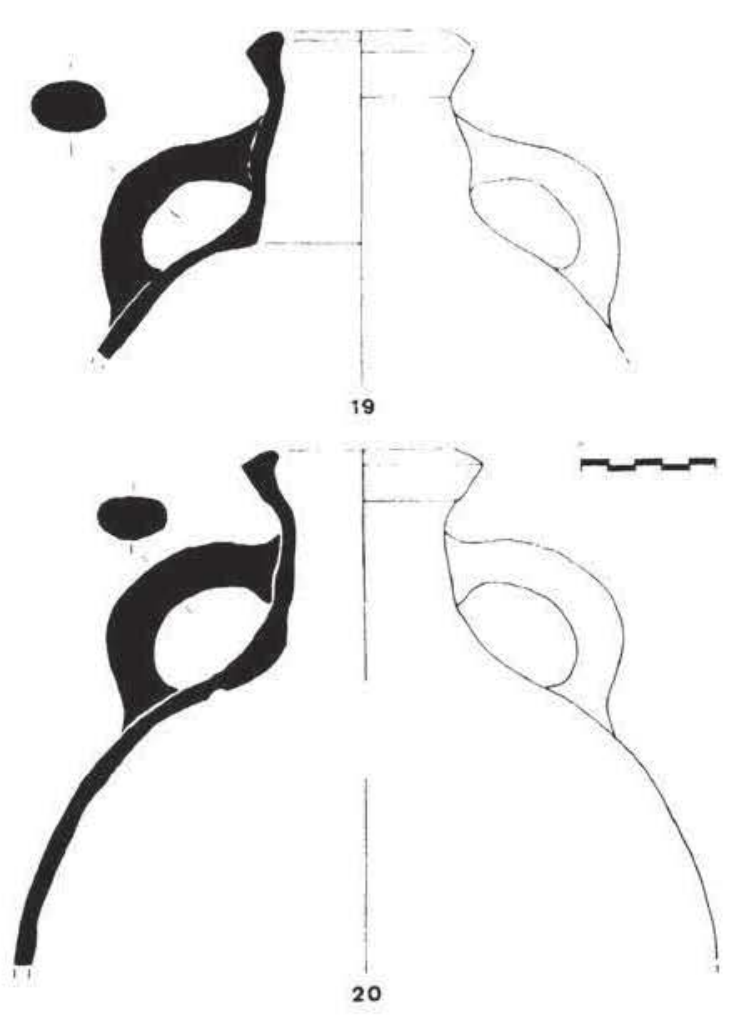

Figura 4, Fragmentos procedentes de la intervención del Hospital Vell de Santa Tecla (1990) [19] y Plaza dels Angels (1986) [20]. E. 1:4.

temente similar, obtenemos un total estimado de ánforas de 18 que, en función de su origen, se distribuyen de la siguiente manera: cuatro de origen oriental $(22,2 \%)$, cuatro de procedencia sudhispánica $(22,2 \%)$, cuatro originarias del Norte de Africa $(22,2 \%)$ y seis de procedencia no determinada $(33,3 \%$ ) (figura 7 ). El tipo VLR 8.198 está representado con dos ejemplares estimados $(11,1 \%)$, el porcentaje más alto, sobre una muestra muy reducida, de los obtenidos hasta ahora.

\section{INVENTARIO}

20. Parte superior. Número de registro: $86 \mathrm{M}-224-863 / 870 / 887 / 888$. Diámetro de abertura de la boca: $6,6 \mathrm{~cm}$. Presenta restos de un titulus pictus fragmentario y deteriorado sobre el cuello y los hombros (Figura 4). 
21. Parte superior. Número de registro: $86 \mathrm{M}-224-864 / 865 / 889 / 867 / 872$. Diámetro de abertura de la boca: $6,6 \mathrm{~cm}$ (Figura 5 ).

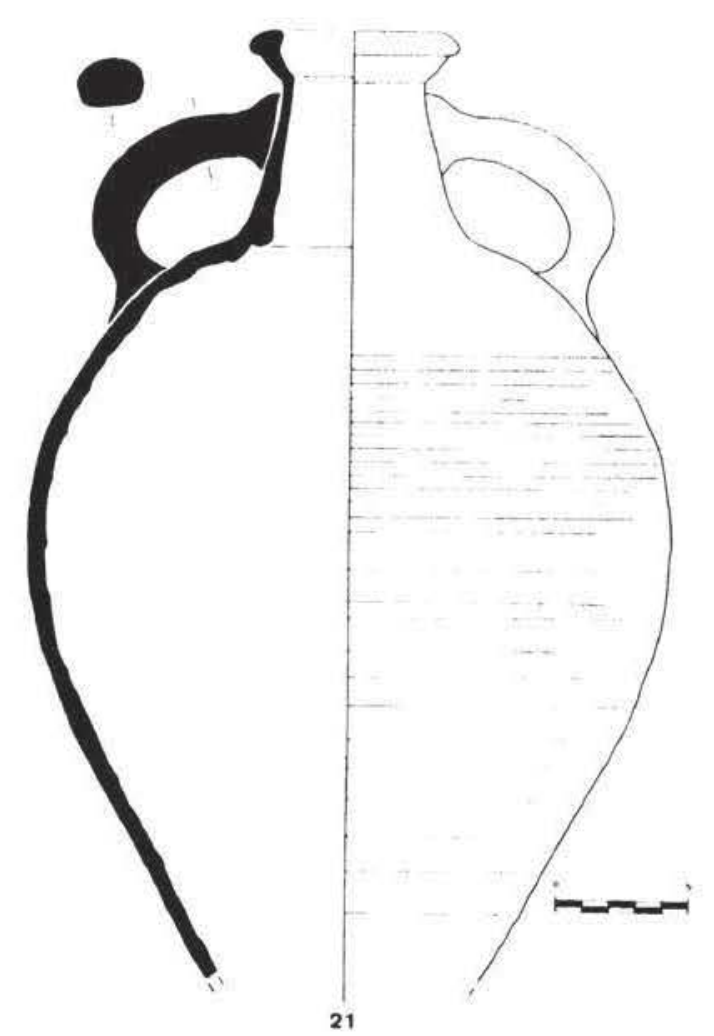

Figura 5.-Fragmentos procedentes de la intervención en la Plaza dels Angels (1986) [21]. E. 1:4.

\section{ANTIGA AUDIĖNCIA, 1986}

En 1986, se realizó una campaña de excavaciones en el edificio de la Antigua Audiencia Provincial de Tarragona ${ }^{6}$, para la construcción del cual se habían reaprovechado parte de las estructuras correspondientes a un complejo sistema de accesos, situado en el ángulo occidental del foro provincial, que comunicaba el exterior del recinto forense con la plaza de representación (Aquilué, 1992, 283) (figura 1). La fase tardo-romana estaba formada, básicamente, por los niveles de amortización de las estructuras alto-imperiales y una escombrera

" Excavación dirigida per J.M. Carreté y X. Dupré, a quienes agradecemos haber puesto a nuestra disposición el material. excavada en los rellenos constructivos (UE 1077. $1084,1075,1087,1038,1085,1072,1071$ y 1065). El estudio de la cerámica de importación de la escombrera, de donde procede el conjunto de materiales más importante, otorga a esta fase una cronologia de segunda mitad avanzada del siglo $\mathrm{v}$, con toda probabilidad en el último cuarto de esta centuria (Aquilué, 1992, 319).

Por lo que se refiere a las ánforas, el total estimado de ejemplares es de 50, los cuales se distribuyen, en función de su zona de procedencia, de la siguiente manera: 17 tienen su origen en el Mediterráneo Oriental (34\%), 12 son de procedencia africana $(24 \%), 10$ son originarios del Sur de Hispania $(20 \%)$ y los 11 restantes son de origen no determinado $(22 \%)$ (figura 7). El tipo VLR 8.198 está representado con un único ejemplar, identificado a partir de fragmentos de pared que no se incluyen en el inventario ( $2 \%$ sobre el total de ánforas).

\section{CONCLUSIONES}

Los 21 fragmentos que presentamos en el inventario, a pesar de lo reducido de la muestra, inducen a plantear una serie de consideraciones acerca de este tipo de ánfora tardo-romana. Los materiales analizados proceden de cinco escombreras tardo-romanas localizadas en la parte alta de Tarragona, lugar ocupado en época alto-imperial por un gran complejo urbanistico vinculado a la administración provincial, que comprende un área sacra en torno al templo dedicado al culto imperial, una gran plaza porticada utilizada como espacio de representación y un circo que separa el recinto provincial de la ciudad propiamente dicha (TED'A, 1989 b) (figural). La cronología de los cinco depósitos se sitúa dentro del siglo $v$ d. C. (los contextos analizados se sitúan entre el segundo y tercer cuarto de este siglo), momento en el cual los antiguos espacios públicos se transforman en áreas de habitación.

El volumen total de individuos anfóricos estimados es de $606^{7}, 16$ de los cuales se atri-

\footnotetext{
7 Para esta valoración cuantitativa se han tenido en consideración los siguientes datos: Vila-roma 1987 (400 ejemplares estimados correspondientes a la UE 918), Trin-
} 
buyen al tipo VLR $8.198(2,6 \%)$. Un indice de presencia bajo, pero superior, en estos contextos, al que presentan otros tipos como la Late Roman Amphora 2/Keay 65 o el tipo Agora de Atenas M.273, que representan el $0,5 \%$ y el $0,7 \%$ respectivamente, en la escombrera de Vila-roma (1987). La distribución por áreas de producción de los 606 ejemplares estimados es la siguiente: 159 originarios del Mediterráneo Oriental $(26,23 \%), 153$ procedentes del Norte de Africa $(25,24 \%), 136$ de origen sudhispánico $(22,44 \%)$, 27 posiblemente producidas en el área tarraconense $(4,45 \%)$ y 132 de procedencia indeterminada $(21,78 \%$ ) (figura 7 ).

La estructura completa del tipo, restituible parcialmente a partir del ejemplar número 21 (figura 5), es de forma piriforme con base apuntada diferenciada, cuerpo ligeramente acanalado por debajo del arranque inferior de las asas, cuello tronco-cónico y borde generalmente exvasado y engrosado al exterior. La sección del borde es, a pesar de ciertas variaciones, resultado de la inflexión hacia el interior del labio con perfil pseudo-triangular (ejemplares 3, 4, $9,10,11,12,17,19$ y 20) o, en menor proporción, engrosado y redondeado ( 5 y 21 ). La inflexión del labio crea una superficie interior cóncava $y$, exteriormente, la base del borde aparece generalmente indicada por un pequeño resalte. El diámetro de abertura de la boca de los ejemplares presentados oscila entre 5,6 y $6,6 \mathrm{cms}^{x}$. A pesar de las diferencias que se

quet Vell-4 1989 (93 ejemplares estimados correspondientes a las UE 106, $110,111,113$ y 114), Hospital Vell de Santa Tecla 1990 (45 ejemplares estimados correspondientes a la UE 109), Plaza dels Angels 1986 (18 ejemplares estimados correspondientes a las UE 223 y 224) y Antiga Audiència ( 50 ejemplares estimados correspondientes a las UE 1084, 1077, 1075, 1087, 1038, 1085, 1072, 1071 y 1065). Los cinco ejemplares recuperados en la intervención del año 1986 en la escombrera de Vila-roma y que se incluyen en el inventario, no se incorporan a la cuantificación ya que se encuentran en fase de estudio. El criterio utilizado en la estimación de los ejemplares se basa en el número mínimo de individuos representados a partir de fragmentos indicativos de unidades diferentes. Cuando la presencia de un tipo se ha constatado únicamente mediante fragmentos de pared, $y$ no ha sido posible establecer sin lugar a dudas su pertinencia a individuos diferentes, se ha considerado el número minimo de ejemplares.

* Existen ejemplares de diámetro más amplio como el publicado por M. Bonifay (1987, fig. 9-35) que presenta una abertura de $8,5 \mathrm{~cm}$. aprecian tanto en el tratamiento del borde como en otros detalles de la estructura del ánfora, to limitado de la muestra nos induce a rehuir cualquier intento de definir variantes que, sobre la base actual disponible, han de atribuirse más a la casuistica de la elaboración de la pieza que a motivaciones de carácter productivo y/o cronológico. El cuello presenta las paredes perpendiculares o moderadamente reentrantes y su unión con el cuerpo presenta una acentuada inflexión reflejada por un resalte interior. Las asas son de sección ovalada y se unen al cuello por debajo del borde y al inicio de la unión de éste con el cuerpo.

Técnicamente, existe una gran uniformidad aparente, tanto en la coloración como en la textura y composición de los fragmentos analizados. Las pastas presentan coloraciones entre beige y marrón, con tonalidades anaranjadas, están bien depuradas y presentan la inclusión de partículas de cal (en algunos casos «rompiendo» la superficie) y mica, generalmente en proporciones muy bajas. Esta homogeneidad aparente se ve confirmada por los resultados de los análisis por florescencia de rayos-X obtenidos sobre las cuatro muestras procedentes de ejemplares recuperados en la escombrera de Vila-roma ( $v$. supra). Sin embargo, si que podemos apuntar pequeñas diferencias aparentes en los dos ejemplares procedentes de la plaza dels Angels 1986 (ejemplares 20 y 21), que presentan pastas más porosas y blandas, coloración más oscura y mayor presencia de particulas de mica. Ignoramos si estas diferencias pueden, en parte, atribuirse, dada la uniformidad general, a alteraciones producidas por el entorno sedimentológico en el cual se encontraban o si bien han de atribuirse a variaciones productivas.

Para finalizar este apartado descriptivo, hay que señalar que algunos ejemplares presentan sobre el cuello y los hombros restos de tituli picti en tinta roja (ejemplares $2,13,15$ y 20). Es dificil valorar el porcentaje real de individuos con esta característica, dado el alto grado de fragmentación de las piezas $y$, especialmente, la extraordinaria fragilidad de la tinta que, en muchos casos, ha desaparecido o su presencia puede tan sólo intuirse. Los caracteres pinta- 


\begin{tabular}{|l|l|l|l|l|l|l|l|l|l||}
\hline NUM. REG. & Fe $\mathrm{O}_{3}$ & $\mathrm{Mn} \mathrm{O}$ & $\mathrm{Ti} \mathrm{O} \mathrm{O}_{2}$ & $\mathrm{Ca} \mathrm{O}$ & $\mathrm{K}_{2} \mathrm{O}$ & $\mathrm{Si} \mathrm{O} \mathrm{O}_{2}$ & $\mathrm{Al}_{2} \mathrm{O}_{3}$ & $\mathrm{P}_{2} \mathrm{O}_{5}$ & $\mathrm{Mg} \mathrm{O}$ \\
\hline VLR-2979 & 5,61 & 0,09 & 0,69 & 11,35 & 2,45 & 49,45 & 13,23 & 0,16 & 1,57 \\
\hline VLR-2976 & 5,58 & 0,10 & 0,69 & 11,81 & 2,54 & 50,29 & 12,97 & 0,16 & 1,36 \\
\hline VLR-2985 & 5,18 & 0,09 & 0,68 & 11,36 & 2,70 & 47,34 & 12,78 & 0,15 & 1,45 \\
\hline VLR-2977 & 5,41 & 0,08 & 0,72 & 9,67 & 2,66 & 50,69 & 13,73 & 0,16 & 1,47 \\
\hline
\end{tabular}

Figura 6. - Tabla del contenido en hierro, manganeso, titanio, calcio, potasio, silicio, aluminio, fösforo y magnesio de las cuatro muestras de ánfora VL.R 8.198, procedentes de Vila-roma (1987), analizadas por florescencia de rayos-X.

dos parecen similares a los que presentan, en especial, ejemplares de ánfora oriental pertenecientes al tipo LRA I/Keay LIII, indicando, generalmente, conceptos de tipo comercial.

Por lo que respecta a otros ejemplares publicados pertenecientes a este tipo, hemos de reseñar que en nuestra publicación de 1989 (Remolà/Abelló, 304-305) incluiamos como posibles paralelos, un fragmento de borde procedente de la Schola Praeconum de Roma, datado entre el 425-450 d. C. (Whitehouse et alii, 1982, fig. 13-191), cuyas características tipológicas y técnicas parecían acomodarse al tipo VLR $8.198^{\circ}$. En la actualidad, consideramos dudosa la inclusión de este fragmento dentro del tipo que presentamos, sin realizar un análisis más profundo. Esta duda no existe en el caso del ejemplar publicado, de los dos que aparecieron, por M. Bonifay procedente del periodo 1 de La Bourse (Marsella), datado en el segundo cuarto/mediados del siglo $v$ d. C. $(1987,284$, fig. 9-35), el cual reúne todas las características tipológicas y técnicas propias del tipo ${ }^{10}$.

\footnotetext{
La descripción de la pasta parece similar a la que presentan los ejemplares del tipo VLR 8.198: "[Fabric 26:] Smooth and fairly hard with light red $(2.5$ YR $6 / 6)$ to reddish yellow (7.5 YR 7/6) cores and pink (5-7.5 YR 7/4) surfaces. Most sherds contain mica, some are sandy, but there are few other large inclusions. 8 per cent ( 10 per cent by weight) have very pale brown (10 YR 8/3) slip on the outside.n.

10 "Pâte chamois à strates brun foncé, très fine et compacte, légère, avec de nombreuses petits inclusions noires. quelques inclusions blanques; surface chamois ou beigeorangé, douce au toucher, très légèrement micacée.n Uno de los dos ejemplares recuperados presentaba restos fragmentarios de un titulus pictus pendiente de estudio.
}

También se documenta un ejemplar asimilable a este tipo entre el material recuperado en la villa romana de Puig Rodon (Baix Empordà) (Nolla/Casas, 1990, 208-209, fig. 17) ". El ejemplar en cuestión procede de la UE 2021 datada dentro de la primera mitad/mediados del siglo v. En la restante bibliografia consultada no hemos podido identificar ningún ejemplar que pudiera ser atribuido con cierta seguridad a este tipo ${ }^{12}$.

Finalmente, quedan sin resolver temas tan importantes como el origen y el contenido de este tipo. Respecto al primer punto, y con todas las matizaciones al uso, consideraciones de tipo técnico y tipológico, asi como la presencia relativamente alta de tituli picti (más usual, aunque no exclusivo, de recipientes anfóricos

\footnotetext{
1 Agradecemos la inestimable colaboración de J. M. Nolla y J. Casas.

12 Véase capitulo de bibliografia en TED'A 1989, al cual hay que añadir: Crowfoot. J. W.. Fitzgerald, G. M., 1929. Excavations in the Tyropoeon Valley, Jerusalem. Annual of the Palestine Exploration Fund 5 (1927), Manchester. 1929. Tubb, J. N., 1986, The pottery from a Byzantine well near Tell Fara, en Palestine Exploration Quarterly, January-June 1986. Williams, C., 1977, A Byzantine welldeposit from Anemurium (Rough Cilicia), en Anatolian Studies, vol. XXVII (1977), 175-190. Zemer, A., 1977. Storage jars in Ancient Sea Trade, Haifa. Diez, F., Cerámica común romana de la Galilea. Madrid, 1983. Berato, J., Borreani, M., Lacacheur, P., Pasqualini, M., Theveny, J-M., Rigoir, J. et Y., 1986, Fouilles récentes à Toulon (Var), en Documents d'Archéologie Meridionale 9, 1986. 135-166. Kelemen, M. H., Roman amphorae in Pannonia, en Acta Archaeologica, XXXIX, 1987, 1-86. Arthur, P.. Some observations on the economy of Bruttium under the Later Roman Empire, en Journal of Roman Archaeology, 2. $1989,133-141$.
} 


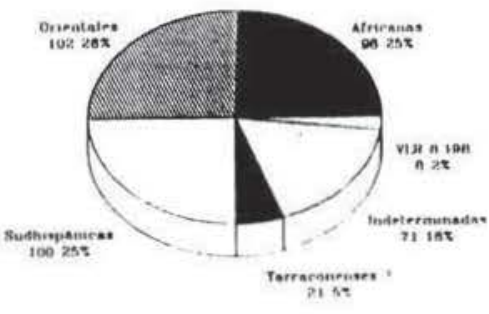

1

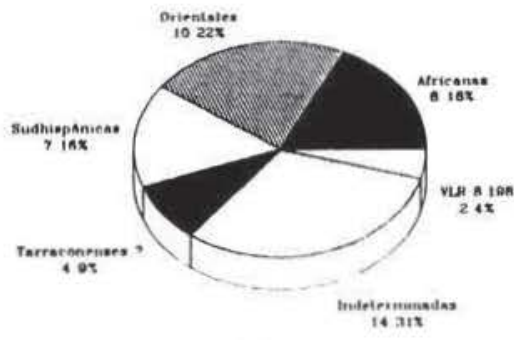

3

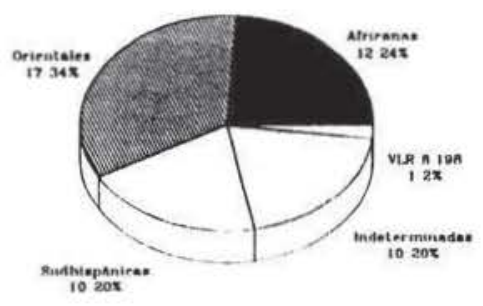

s

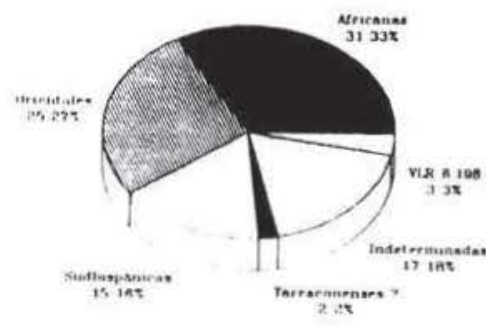

2
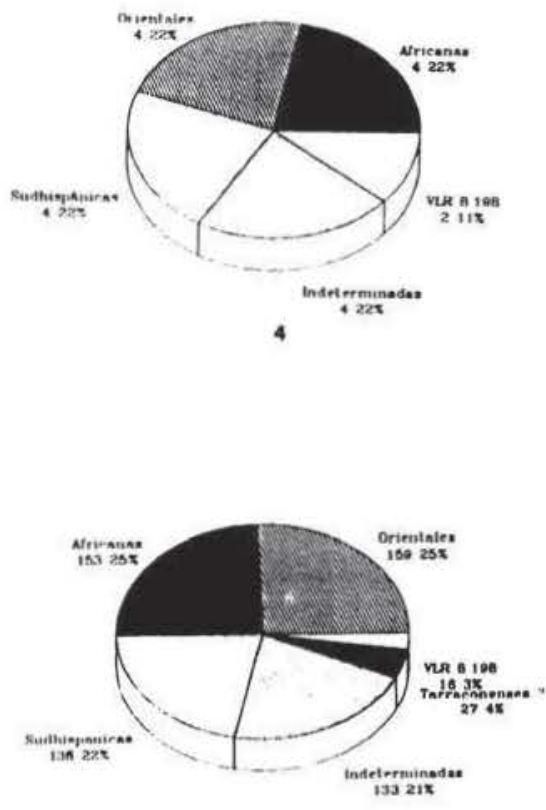

6

Figura 7.-Gráficos por áreas de procedencia: [1] Vila-roma (1987), [2] Trinquet Vell-4, [3] Hospital Vell de Santa Tecla, [4] Plaza dels Angels, [5] Antiga Audiència y [6] Totales.

de este origen), nos inducen a plantear, en espera de argumentos más consistentes y fiables, un posible origen oriental (¿islas egeas?).

\section{BIBLIOGRAFIA}

AQUILUÉ, X., 1992: Las cerámicas africanas procedentes de la Colonia Iulia Urbs Triumphalis Tarraco, Barcelona, gener 1991. Edición microfichada: "Col.lecció de tesis doctorals microfitxades» núm. 1275 (1992).
Bonifay, M., 1987: «Observations sur les amphores à Marseille d'après les fouilles de La Bourse (1980-1984)", en Revue d'Archéologie de Narbonnaise, 16-86 (1987), 269-305.

KeAY, S. J., 1984: Late Roman Amphorae in the Western Mediterranean. A tipology and economic study: the catalan evidence, BAR International Series 196, Oxford, 1984.

Nolla, J. M., y CASAS, J., 1990: «El material ceràmic d'importació de la vil.la romana de Puig Rodon (Corçà, Baix Empordà), d’època 
severiana a la Baixa Antiguitat", en $C Y P$ SELA, VIII. (1990), 193-218.

REMOLA, J. A., y ABFLLó, A., 1989: «Les àmfores", en Un abocador del segle $r d$. C. en el Fórum Provincial de Tàrraco, Memòries d'excavacio 2, Tarragona 1989, 249-320.

TED'A, 1989 a: Un abocador del segle i d. $C$. en el Fòrum Provincial de Tàrraco, Memòries d'excavació 2, Tarragona, 1989.
TED'A, 1989 b: «El foro provincial de Tarraco, un complejo arquitectónico de época flavia", en Archivo Español de Arqueología,62 (1989), 141-191.

Whitehouse, D.; Barker, G.; Refle, R., y Refse, D.. 1982: "The Schola Praeconum I: The coins, Pottery, Lamps and Fauna», en $P a$ pers of the British School at Rome, L (1982), 53-101.

\title{
ÁNFORAS ITÁLICAS DE ÉPOCA TARDORROMANA EN HISPANIA. LOS HALLAZGOS DEL PALMAR HOTEL (PREMIÀ DE MAR, BARCELONA)
}

\author{
POR
}

\author{
RAMÓN COLL i MONTEAGUDO \\ Museu Municipal d’Estampació Tèxtil. \\ Premià de Mar (Barcelona).
}

$\mathrm{Y}$

RAMÓN JÁRREGA DOMÍNGUEZ

Escuela Española de Historia y Arqueologia, C.S.I.C. Roma.

\section{RESUMEN}

En este trabajo estudiamos un nuevo tipo de ánfora recientemente identificado, perteneciente a la época tardorromana. Estas ánforas probablemente transportaban vino, que era usado para abastecer la ciudad de Roma y eventualmente se comercializaba en otros paises del Imperio; al menos, éste es el caso de Hispania, evidensiado por los materiales que aqui estudiamos.

\section{SUMMARY}

In this work, we study a recently known kind of Italian amphora of Late Roman times. These amphorae probably carried wine, used to supply the city of Rome and eventually for trade with other countries of the Empire; at least, this is the case of Hispania, evidenced by the materials studied here.
El estado actual de conocimientos sobre las ánforas tardorromanas ' pone de manifiesto la preponderancia casi absoluta de los productos africanos (en los que se envasaba principalmente vino y salazones) entre finales del siglo $\| \mathrm{y}$ el siglo vı d. C., así como la presencia (menor pero no insignificante) de producciones anfóricas procedentes del Mediterráneo oriental. La citada preponderancia de los productos norteafricanos envasados en ánforas coincide plenamente con el auge comercial de la zona correspondiente a la actual Túnez, que además de por los

\footnotetext{
I Una extensa clasificación tipológica y un pormenorizado estudio de conjunto sobre las ánforas tardorromanas puede verse en Keay 1984 passim.
} 